\title{
GENETIC ANALYSIS OF A POPULATION OF TRIBOLIUM \\ II. METABOLIC PATTERN OF CORN OIL SENSITIVE ANOMALY*
}

\author{
R. F. COSTANTINO, $\dagger$ J. C. ROGLER and A. E. BELL \\ Population Genetics Institute and Department of Animal Sciences, \\ Purdue University, Lafayette, Indiana
}

Received 28.xii.67

\section{INTRODUGTION}

IN earlier papers (Costantino et al., 1966, 1967), two properties of a unique population of Tribolium castaneum were established. Firstly, corn (maize) oil inhibits larval growth and the degree of inhibition is a function of the concentration of corn oil in the diet; and secondly, the genetic basis of this inhibition is an autosomal recessive gene. Furthermore, these two properties accounted for certain unexpected phenotypic changes observed in the growth characteristics of this population during a long-term selection study.

Our attention is now focused on (1) describing the mutant phenotype, and (2) elucidating the biological mechanism(s) responsible for the population's response to corn oil.

\section{Materials AND Methods}

The genetic material for this study consisted of the two populations, corn oil sensitive $(\cos / \cos )$ and Purdue Foundation $(+1+)$, described in our earlier studies and the reciprocal crosses between these two populations.

Highly purified fatty acids (99 per cent. + ), palmitic, stearic, oleic, linoleic, and linolenic, for addition to certain diets were obtained from the Nutritional Biochemicals Corp. of Cleveland, Ohio. The fatty acids were dissolved and made to volume in a solution of 0.05 per cent. hydroquinone in reagent grade diethyl ether. Aliquots of the solution containing the fatty acid were thoroughly combined with the basal diet and the diethyl ether allowed to evaporate. An equal aliquot of the hydroquinone-ether solution without fatty acid was added to the control diet. Other than for prescribed modifications of the diet as described in later sections, all animals were uniformly cultured at $33^{\circ} \mathrm{C}$. and 70 per cent. relative humidity. Standard Tribolium techniques were used for obtaining quantitative measurements.

\section{RESUlts AND DISGUSSION}

\section{(i) Description of mutant phenotype}

Five criteria were used to describe the corn oil sensitive mutant: (1) 13-

* Journal Paper No. 3263 from Purdue University Agricultural Experiment Station. Based in part on a thesis submitted by the senior author in partial fulfilment of the Doctor of Philosophy degree at Purdue University. This study was supported by N.I.H. Training Grant GM-00024, NSF Research Grant G-15824 and USDA Cooperative Agreement 12-14-100-5448(44), Pioneering Research Laboratory for Animal Genetics.

$\uparrow$ Present Address: Department of Biology. The Pennsylvania State University, University Park, Pa. 16802. 
day larval weight; (2) percentage of viability from egg to 13-day larva; (3) developmental time (days to pupation); (4) percentage of viability from 13-day larva to pupa; and (5) percentage of viability from pupa through adult emergence. Two hundred eggs representing each genotype (cos/cos, $+/+$ and reciprocal $F_{1}$ 's) were cultured on each of four diets which were differentiated by percentage of corn oil (table 1). Thirteen days later all viable larvae in each genotype-diet combination were counted, a sample of 100 were individually weighed, and all were returned to their prescribed diets for daily observation as to pupation time and viability through adult emergence.

\section{TABLE 1}

Diets used to describe corn oil sensitive mutant

\begin{tabular}{lrrrr} 
& \multicolumn{4}{c}{ Diet (\% weight) } \\
\cline { 2 - 5 } \multicolumn{1}{c}{ Component } & 0 & 2 & 4 & 6 \\
Whole wheat flour & 89 & 89 & 89 & 89 \\
Dried brewer's yeast & 5 & 5 & 5 & 5 \\
Glucose monohydrate & 6 & 4 & 2 & 0 \\
Corn oil & 0 & 2 & 4 & 6
\end{tabular}

The results as summarised in table 2 show that 13-day larval weight of the cos/cos genotype was reduced as the concentration of corn oil in the diet increased. Heterogeneity of variances among genotype-diet combinations prevented an overall statistical analysis; however, it appears that all genotypes were adversely affected at the highest level of corn oil. Larval weights of the reciprocal $F_{1}$ 's suggest a consistent maternal effect in favour of $+1+$ females. Also, with one exception, the $F_{1}$ 's were heavier than either parent and confirmed the earlier finding of Englert and Bell (1963) regarding the heterotic nature of larval weight in Tribolium. It is of interest to observe that on the diet containing no corn oil larval weight of the cos/cos genotype was normal. Thus this system is somewhat analogous to phenlyketonuria in humans where dietary intake of a bare subsistence level of phenylalanine presumably circumvents the genetic defect. In the case of corn oil sensitivity in Tribolium, it is possible to control and minimise the genetic defect. The analogy is not complete, however, for phenylalanine is required by the phenylketonuric whereas there is no evidence that the beetles require corn oil.

Hatchability and survival of larvae to 13 days of age was unaffected by level of corn oil in the diet of all genotypes other than cos/cos. The latter genotype was adversely affected, especially on diets 4 and 6 .

Further interactions of genotypes with level of corn oil are revealed in table 2. For example, developmental time as measured by days to pupation was essentially equal for the $+1+$ and $\cos / \cos$ genotypes on diet 0 , yet $\cos / \cos$ took an increasing amount of time to reach the pupal stage as the level of oil was increased in the diet, while the $+1+$ genotype was unaffected except possibly at the highest level. Variation in days to pupation increased sharply for the cos/cos genotype on higher levels of oil while the other genotypes remained relatively uniform (see standard errors of appropriate means). Again, the $F_{1}$ populations exhibited a marked heterosis; however, no maternal effects were manifested in developmental time.

The survival to pupation of all genotypes other than cos/cos were unaffected 
by dietary corn oil. Note that the mutant type had excellent viability on diet 0 , but on the higher levels of corn oil viability was adversely affected at all stages of development.

TABle 2

Description of the mutant corn oil sensitive phenotype

\begin{tabular}{|c|c|c|c|c|c|c|}
\hline \multirow{2}{*}{$\begin{array}{l}\text { Diet } \\
(\% \text { corn oil })\end{array}$} & \multirow{2}{*}{ Genotype } & \multicolumn{2}{|c|}{ At 13-days } & \multirow{2}{*}{$\begin{array}{l}\text { Days to } \\
\text { pupation }\end{array}$} & \multicolumn{2}{|c|}{$\%$ viability } \\
\hline & & Larval wt. $(\mu \mathrm{g})$ & $\%$ viability & & pupa & adults \\
\hline 0 & $\begin{array}{l}+1+ \\
\cos /+^{*} \\
+\mid \cos \dagger \\
\cos / \cos \end{array}$ & $\begin{array}{l}2165 \cdot 3 \pm 53 \cdot 5 \\
2500 \cdot 8 \pm 22 \cdot 9 \\
2321 \cdot 4 \pm 37 \cdot 4 \\
2263 \cdot 2 \pm 39 \cdot 3\end{array}$ & $\begin{array}{l}88 \\
91 \\
83 \\
76\end{array}$ & $\begin{array}{l}16 \cdot 7 \pm 0 \cdot 12 \\
16 \cdot 0 \pm 0 \cdot 11 \\
15 \cdot 9 \pm 0 \cdot 13 \\
16 \cdot 4 \pm 0 \cdot 13\end{array}$ & $\begin{array}{r}97 \\
100 \\
95 \\
100\end{array}$ & $\begin{array}{r}100 \\
100 \\
100 \\
99\end{array}$ \\
\hline 2 & $\begin{array}{l}+1+ \\
\cos \mid+ \\
+\mid \cos \\
\cos \mid \cos \end{array}$ & $\begin{array}{l}2314 \cdot 8 \pm 46 \cdot 3 \\
2466 \cdot 1 \pm 30 \cdot 3 \\
2404 \cdot 8 \pm 21 \cdot 4 \\
1634 \cdot 7 \pm 50 \cdot 6\end{array}$ & $\begin{array}{l}90 \\
88 \\
78 \\
68\end{array}$ & $\begin{array}{l}16 \cdot 5 \pm 0 \cdot 13 \\
15 \cdot 9 \pm 0 \cdot 12 \\
15 \cdot 7 \pm 0 \cdot 12 \\
17 \cdot 9 \pm 0 \cdot 15\end{array}$ & $\begin{array}{r}98 \\
100 \\
99 \\
99\end{array}$ & $\begin{array}{r}98 \\
99 \\
100 \\
100\end{array}$ \\
\hline 4 & $\begin{array}{l}+1+ \\
\cos /+ \\
+/ \cos \\
\cos / \cos \end{array}$ & $\begin{array}{r}2322 \cdot 8 \pm 36 \cdot 2 \\
2418 \cdot 4 \pm 23 \cdot 0 \\
2236 \cdot 9 \pm 38 \cdot 5 \\
706 \cdot 2 \pm 55 \cdot 3\end{array}$ & $\begin{array}{l}84 \\
87 \\
86 \\
59\end{array}$ & $\begin{array}{l}16 \cdot 8 \pm 0 \cdot 15 \\
15 \cdot 7 \pm 0 \cdot 11 \\
15 \cdot 9 \pm 0 \cdot 14 \\
21 \cdot 0 \pm 0 \cdot 38\end{array}$ & $\begin{array}{r}100 \\
99 \\
100 \\
90\end{array}$ & $\begin{array}{r}100 \\
99 \\
98 \\
89\end{array}$ \\
\hline 6 & $\begin{array}{l}+1+ \\
\cos /+ \\
+/ \cos \\
\cos / \cos \end{array}$ & $\begin{array}{r}2098 \cdot 4 \pm 43 \cdot 1 \\
2271 \cdot 1 \pm 29 \cdot 2 \\
2120 \cdot 9 \pm 27 \cdot 5 \\
291 \cdot 8 \pm 34 \cdot 0\end{array}$ & $\begin{array}{l}89 \\
88 \\
84 \\
61\end{array}$ & $\begin{array}{l}17 \cdot 3 \pm 0 \cdot 15 \\
16 \cdot 4 \pm 0 \cdot 16 \\
16 \cdot 4 \pm 0 \cdot 15 \\
25 \cdot 1 \pm 0 \cdot 53\end{array}$ & $\begin{array}{l}97 \\
97 \\
98 \\
74\end{array}$ & $\begin{array}{l}79 \\
81 \\
80 \\
69\end{array}$ \\
\hline & & parent $\cos / \cos$ & $\dagger$ male pa & $+1+$ & & \\
\hline
\end{tabular}

An interesting observation is that corn oil as high as 6 per cent. of the diet appears to have no effect on larval and pupal viability of all genotypes other than $\cos / \cos$, yet adult viability for all genotypes was reduced some 15-20 per cent. on diet 6 . The latter metamorphic inhibition by 6 per cent. corn oil affected all genotypes and appears unrelated to the cos genetic anomaly.

Prior to initiating studies utilising the components of corn oil, it appeared desirable to determine whether cos action was continuous throughout growth or whether the defect could be circumvented by early avoidance. To resolve this question, cos/cos larvae were grown initially on a diet devoid of supplemental corn oil for $2,4,6,8$, and 10 days and were then placed on a diet containing 5 per cent. corn oil. The results as summarised in table 3 indicate that the genetic defect was not circumvented during larval growth. However, it is apparent that the length of time the larvae were grown on the corn oil unsupplemented diet altered the expressivity of cos. This suggested that the cos gene may be hypomorphic with respect to the utilisation of this oil and that perhaps the accumulation of a component of corn oil or deleterious metabolic products over time as the oil was ingested was responsible for the observed growth inhibition.

\section{(ii) Hypothesis of gene action}

When other vegetable oils (cottonseed, soybean, olive and hydrogenated coconut) were tested for their inhibitory effect on the cos genotype, it was 
found that corn oil was not unique. Other oils inhibited the growth of $\cos / \cos$ and the amount of inhibition was positively correlated with the degree of unsaturation of the oils.

TABLE 3

Phenotypic expression of $\cos / \cos$ on a corn oil unsupplemented diet for increasing periods before switching to the corn oil supplemented diet

$\begin{array}{cccc}\begin{array}{c}\text { Corn oil } \\ \text { treatment } \\ \text { combination }\end{array} & \begin{array}{c}\text { Days initially } \\ \text { without } \\ \text { corn oil* }\end{array} & \begin{array}{c}\text { Subsequent days } \\ \text { with } \\ \text { corn oil* }\end{array} & \begin{array}{c}\text { Mean 13-day } \\ \text { larval weight } \\ (\mu \mathrm{g})(n=50)\end{array} \\ 1 & 0 & 13 & 190 \pm 26 \\ 2 & 2 & 11 & 209 \pm 28 \\ 3 & 4 & 9 & 231 \pm 38 \\ 4 & 6 & 7 & 290 \pm 26 \\ 5 & 8 & 5 & 506 \pm 24 \\ 6 & 10 & 3 & 612 \pm 32 \\ 7 & 13 & 0 & 742 \pm 48\end{array}$

* Common ingredients were corn meal 50 per cent., soybean meal 17 per cent., vitamin premix 10 per cent., and dried brewer's yeast 10 per cent. The corn oil unsupplemented diet had 5 per cent. glucose monohydrate substituted for 5 per cent. corn oil of the supplemented diet.

The approximate composition of corn oil is $98 \cdot 6$ per cent. triglycerides, 1.4 per cent. unsaponifiable matter (e.g. sitosterol, tocopherols), 0.3 per cent. free fatty acids and 0.001 per cent. phosphorus. Since the triglycerides predominate and because of the suggested relationship of growth to degree of unsaturation of the fatty acids, an experiment was designed to study the effects of various free fatty acids (palmitic, stearic, oleic, linoleic, linolenic) on growth of $\cos / \cos$ and $+/+$ genotypes. The results are summarised in table 4.

TABLE 4

Mean 13-day larval weight of the $+1+$ and $\cos / \cos$ genotypes grown on diets differentiated by their free fatty acids

\begin{tabular}{lrr}
\multicolumn{1}{c}{ Diets* } & \multicolumn{2}{c}{$\begin{array}{c}\text { Mean weights }(\mu \mathrm{g}) \\
\text { by genotypes }(n=100)\end{array}$} \\
\cline { 2 - 3 } & \multicolumn{1}{c}{$\cos / \cos$} & $+/+$ \\
Control & $1636 \pm 39$ & $2332 \pm 39$ \\
Palmitic & $1834 \pm 50$ & $2466 \pm 28$ \\
Stearic & $2200 \pm 24$ & $2536 \pm 32$ \\
Oleic & $1396 \pm 51$ & $2380 \pm 30$ \\
Linoleic & $400 \pm 21$ & $2496 \pm 19$ \\
Linolenic & $81 \pm 11$ & $2226 \pm 45$
\end{tabular}

* Common ingredients were corn meal 58 per cent., soybean meal 17 per cent., vitamin premix 10 per cent., dried brewer's yeast 10 per cent. and hydroquinone 0.025 per cent. The remainder of the Control Diet consisted of 4.975 per cent. glucose monohydrate. Each experimental diet had $2 \cdot 5$ per cent. of the glucose replaced with the specified free fatty acid. 
The wild type $(+1+)$ genotype was essentially unaffected by the various fatty acids; however, cos/cos showed a profound response. Larval weight of the sensitive genotype increased slightly on the saturated fatty acids, palmitic and stearic, and decreased on the unsaturated fatty acids. This experiment indicated that the magnitude of the larval growth inhibition of the cos homozygote was a function of the degree of unsaturation of the fatty acid present in the diet. Furthermore, corn oil is not a unique dietary inhibitor which makes the gene symbol somewhat misleading. Corn oil is high (58 per cent.) in the unsaturated fatty acid linoleic and this probably accounts for the cos homozygote's response to this oil.

Larval growth of the cos/cos genotype appears to have been inhibited on the control diet of table 4 if one compares it to that of the $+1+$ genotype. Yet the two genotypes had similar 13-day larval weights on the control diet of table 2. This apparent contradiction more than likely arises from the nature of the two diets. Chemical analyses revealed 1.24 per cent. oil (ether extract) in diet 0 of table 2 while the control diet of table 4 contained 3.69 per cent. oil. In fact, the results in table 2 themselves show that the latter level (3.69 per cent.) of corn oil would be sufficiently high to reduce significantly the 13-day larval weight of $\cos / \cos$, and the ether extract in the control diet of table 4 would consist primarily of corn oil.

The above series of experiments led to a quantitative study of the response of the $+1+$ and $\cos / \cos$ genotypes to the following two variables: (1) concentration of fatty acid added to the diet $\left(X_{1}=0,1.25\right.$ per cent. or 2.50 per cent.) and (2) degree of unsaturation of the fatty acid $\left(X_{2}=\right.$ stearic, oleic, linoleic, or linolenic). These fatty acids were chosen because they all have the same chain length (18 carbons) and differ only in degree of unsaturation. The upper level of fatty acid approximates the amount of linoleic acid contained in 5 per cent. corn oil. The composition of the diets are identical to those of table 4 (with the addition of the 1.25 per cent. level for each fatty acid). Fifty larvae were weighed for each concentration-fatty acid combination and the experiment was replicated. The data were analysed using multiple linear regression techniques and the results are shown in fig. 1.

The regression equations given beneath the diagram for each replication clearly reflect the differential response of these two genotypes. For the $+1+$ genotype no regression was found in replication 1 and the linear equation for replication 2 (significant at 0.05 level of probability) indicates larval weight increased as the concentration of fatty acid increased irrespective of the particular fatty acid considered. The response of the cos/cos genotype was different; in both replications the mean larval weight was a function of both concentration and kind of fatty acid. The regression equations had multiple correlation coefficients of 0.978 for replication 1 and 0.963 for replication 2; the overall fit of the data was significant at the 0.01 level of probability in both cases. It is apparent that larval growth of normal $(+1+) T$. castaneum is affected little, if at all, by either these concentrations of free fatty acids or their degrees of unsaturation. Yet the cos/cos genotype is drastically affected by both variables.

The above results are consistent with the hypothesis that the biological function of the cos gene reduces the utilisation of the unsaturated fatty acids. Inhibition of growth then becomes a function of the concentration of the unsaturated fatty acids in the insect's tissues and the degree of unsaturation of the fatty acids involved. 
REPLICATION 1

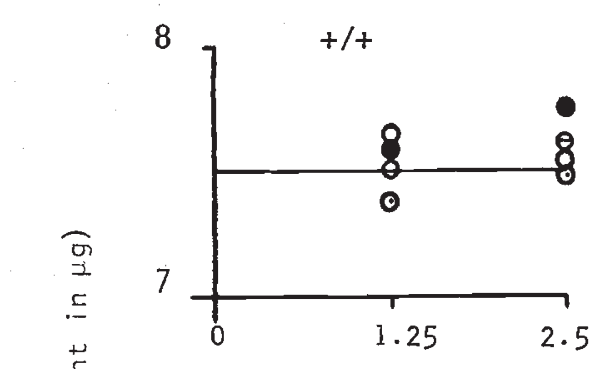

$\cos / \cos$

z

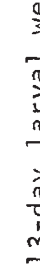

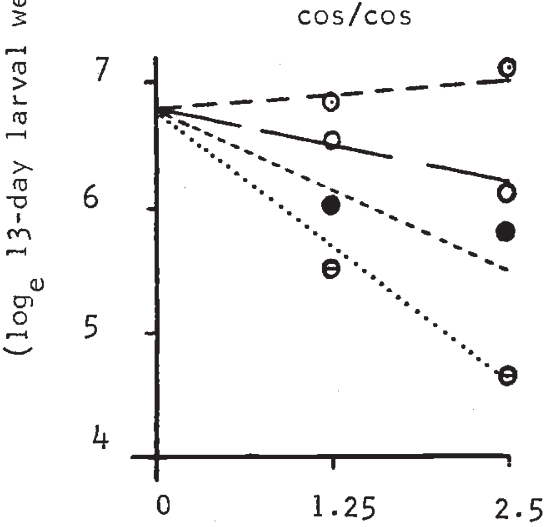

REPLICATION 2

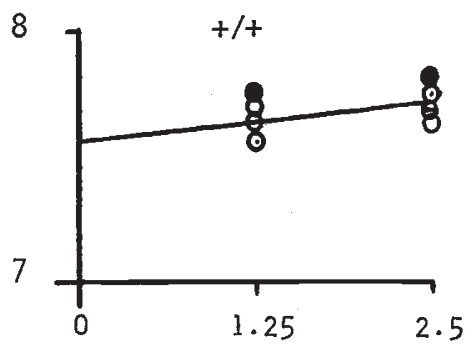

$\cos / \cos$

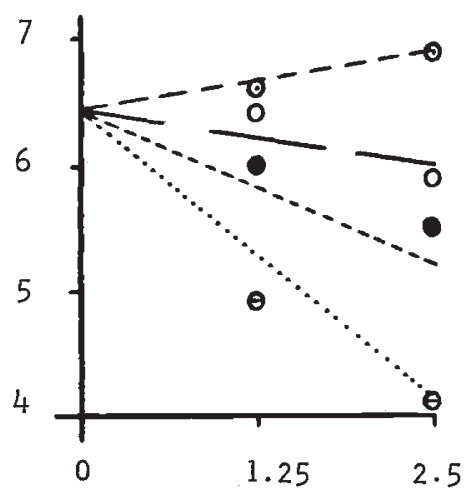

CONCENTRATION OF FATTY ACID

(percent of diet by weight)

$$
\begin{array}{ll}
a_{+}=7.5 & a_{+}=7.6+0.06 X_{1} \\
a_{\cos }=6.8+0.40 X_{1}-0.32 X_{1} X_{2} & a_{\cos }=6.5+0.55 X_{1}-0.37 X_{1} X_{2}
\end{array}
$$

Fig. 1.-Mean response of the $+1+$ and cos/cos genotypes to concentration $\left(X_{1}\right)$ and degree of unsaturation $\left(X_{2}\right)$ of the fatty acids stearic $(\odot)$, oleic $(O)$, linoleic $(\Theta)$, and linolenic $(\Theta)$.

\section{Summary}

1. The corn oil sensitive genotype (cos/cos) of Tribolium castaneum was compared with the normal $(+\mid+)$, and reciprocal $F_{1}$ genotypes in four environments of varying corn oil concentration.

2. The criteria for comparison were (1) 13-day larval weight, (2) hatchability and larval viability to 13 days, (3) developmental time, (4) viability from 13-day larva to pupa and (5) viability from pupa through adult emergence.

3. The cos homozygote showed a drastic change, not apparent in the other genotypes, in each of the criteria as the level of corn oil increased in the environment. The cos/cos genotype was, however, essentially equivalent to the other genotypes when no corn oil was added to the diet.

4. The genetic defect was not circumvented by early larval growth on the diet without supplemental corn oil.

5. Experiments designed to test the influence of the free fatty acids 
palmitic, stearic, oleic, linoleic and linolenic on the 13-day larval weight of the $+/+$ and cos/cos genotypes revealed that (1) the $+1+$ genotype was unaffected by the various fatty acids and (2) larval growth of cos/cos was inhibited and the degree of inhibition was a function of the concentration of the unsaturated fatty acid in the diet and the degree of unsaturation of the fatty acids involved.

6. Growth was not inhibited and appeared to be enhanced by the saturated fatty acids.

\section{REFERENCES}

COSTANTINO, R. F., BELL, A. E., AND ROGLER, J. C. 1966. Genetic control of lipid metabolism in Tribolium. Nature, 210, 221-222.

COSTANTiNo, R. F., BELL, A. E., AND ROGLeR, J. C. 1967. Genetic analysis of a population of Tribolium: I. Corn oil sensitivity and selection response. Heredity, 22, 529 539,

ENGLERT, D. G., AND BELL, A. E. 1963. Genetic differences in the growth curve of Tribolium castaneum. Growth, 27, 87-99. 